\title{
Die guten Vorsätze
}

Die Definition von Meilensteinen ist ein Begriff aus dem Projektmanagement. Meilensteine teilen einen Projektverlauf in Etappen, es sind Zwischenziele, die einen Fortschritt markieren. Nichts anderes sind eigentlich auch die guten Vorsätze für das neue Jahr. Auch wir möchten diese Tradition zum Jahreswechsel begehen und beschäftigen uns deshalb in dieser Ausgabe mit Meilensteinen, die in diesem Jahr in Forschung und Industrie erreicht wurden, und mit neuen Meilensteinen für das Jahr 2021 und darüber hinaus.

In unserem aktuellen ExpertenForum besprechen wir dieses Mal das Thema Perowskit-Solarzellen. Hier wurde erst in diesem Jahr ein neuer Meilenstein erreicht. Mit einer Perowskit-Tandemsolarzelle hält das Helmholtz-Zentrum Berlin einen neuen weltweiten Effizienzrekord. Wir sprachen mit zwei Experten über einen zukünftigen Meilenstein: die Markteinführung der PerowskitSolarzelle als Alternative zur herkömmlichen Siliziumzelle.

Auch in der additiven Fertigung sind in 2020 Fortschritte zu verzeichnen. Wie das Titelbild dieser Ausgabe zeigt, ist es erstmals gelungen, ein
Wohnhaus aus dem Drucker zu erzeugen. Das ist ein spannendes Pilotprojekt, mit dem sich das Verfahren zukünftig als neue Bautechnik etablieren könnte. Mit dem ,Multi Material Jetting können außerdem seit neuestem auch Teile aus mehreren Materialien wie Keramiken und Metallen in einem einzigen Druckvorgang erschaffen werden. Das ermöglicht eine präzise und individuelle Fertigung hochfunktionaler Bauteile.

Wir berichten auch über Meilensteine, die Unternehmen in der Keramikbranche in diesem Jahr erreicht haben. Steuler hat im Sommer einen neuen, hochmodernen Tunnelofen in Höhr-Grenzhausen in Betrieb genommen. Ein solches Ereignis gibt es nur alle paar Jahrzehnte, ein Meilenstein in der Firmengeschichte. Auch Ceramica Mayor, einer der größten Hersteller stranggepresster Keramik in Spanien, hat gemeinsam mit der Maschinenfabrik Gustav Eirich seine Misch- und Dosiersysteme modernisiert und so seine Produktion bereit für die Zukunft gemacht.

Was sind Ihre Meilensteine für das Jahr 2021?

\section{Folgen Sie uns auf Linkedln}

Auf unserer Linkedln-Seite informieren wir Sie über aktuelle Ergebnisse aus der Forschung, Fachveranstaltungen, aktuelle Fachliteratur, unsere neue Expertenfrage und die Inhalte der kommenden Ausgabe der Keramischen Zeitschrift. www.linkedin.com/company/interceram 